\title{
Dynamic Behavior of Positive Solutions for a Leslie Predator-Prey System with Mutual Interference and Feedback Controls
}

\author{
Cong Zhang, Nan-jing Huang, and Chuan-xian Deng \\ Department of Mathematics, Sichuan University, Chengdu, Sichuan 610064, China \\ Correspondence should be addressed to Chuan-xian Deng; dengchx@scu.edu.cn
}

Received 14 August 2013; Accepted 7 November 2013; Published 20 January 2014

Academic Editors: A. Bellouquid, Y. Cheng, and X. Song

Copyright (C) 2014 Cong Zhang et al. This is an open access article distributed under the Creative Commons Attribution License, which permits unrestricted use, distribution, and reproduction in any medium, provided the original work is properly cited.

We consider a Leslie predator-prey system with mutual interference and feedback controls. For general nonautonomous case, by using differential inequality theory and constructing a suitable Lyapunov functional, we obtain some sufficient conditions which guarantee the permanence and the global attractivity of the system. For the periodic case, we obtain some sufficient conditions which guarantee the existence, uniqueness, and stability of a positive periodic solution.

\section{Introduction}

Leslie [1] introduced the famous Leslie predator-prey system

$$
\begin{gathered}
\dot{x}(t)=x(t)[a-b x(t)]-P(x) y(t), \\
\dot{y}(t)=y(t)\left[e-f \frac{y(t)}{x(t)}\right],
\end{gathered}
$$

where $x(t)$ and $y(t)$ stand for the population (the density) of the prey and the predator at time $t$, respectively, and $P(x)$ is the so-called predator functional response to prey.

In system (1), it has been assumed that the prey grows logistically with growth rate $a$ and carries capacity $a / b$ in the absence of predation. The predator consumes the prey according to the functional response $P(x)$ and grows logistically with growth rate $e$ and carrying capacity $x / f$ proportional to the population size of the prey (or prey abundance). The parameter $f$ is a measure of the food quality that the prey provides and converted to predator birth. Leslie introduced a predator-prey model, where the carrying capacity of the predator's environment is proportional to the number of prey, and still stressed the fact that there are upper limits to the rates of increasing of both prey $x$ and predator $y$, which are not recognized in the Lotka-Volterra model. These upper limits can be approached under favorable conditions: for the predators, when the number of prey per predator is large; for the prey, when the number of predators (and perhaps the number of prey also) is small [2].

In population dynamics, the functional response refers to the numbers eaten per predator per unit time as a function of prey density. Kooij and Zegeling [3] and Sugie et al. [4] considered the following functional response:

$$
P(x)=\frac{c x^{p}}{k+x^{p}} \quad(k>0, c, p \text { are positive constants }) .
$$

In fact, the functional response with $p \geq 1$ has been suggested in [4].

It is easy to see that system (1) can be written as the following system:

$$
\begin{gathered}
\dot{x}(t)=x(t)[a-b x(t)]-\frac{c x^{p}(t) y(t)}{k+x^{p}}, \\
\dot{y}(t)=y(t)\left[e-f \frac{y(t)}{x(t)}\right] .
\end{gathered}
$$

When $p=1$ or $p=2$ in system (3), the Leslie system (3) can be written as the following system:

$$
\begin{gathered}
\dot{x}(t)=x(t)[a-b x(t)]-\frac{c x(t) y(t)}{k+x}, \\
\dot{y}(t)=y(t)\left[e-f \frac{y(t)}{x(t)}\right]
\end{gathered}
$$


or

$$
\begin{gathered}
\dot{x}(t)=x(t)[a-b x(t)]-\frac{c x^{2}(t) y(t)}{k+x^{2}(t)}, \\
\dot{y}(t)=y(t)\left[e-f \frac{y(t)}{x(t)}\right],
\end{gathered}
$$

which were considered in [5].

The mutual interference between predators and prey was introduced by Hassell in 1971 [6]. During his research of the capturing behavior between hosts and parasites, he found that the hosts or parasites had the tendency to leave from each other when they met, which interfered with the hosts capturing effects. It is obvious that the mutual interference will be stronger while the size of the parasite becomes larger. Thus, Pan [7] studied a Leslie predator-prey system with mutual interference constant $m$ :

$$
\begin{gathered}
\dot{x}(t)=x(t)[a(t)-b(t) x(t)]-a(t) x(t) y^{m}(t), \\
\dot{y}(t)=y(t)\left[d(t)-e(t) \frac{y(t)}{x(t)}\right],
\end{gathered}
$$

where $a(t), b(t), d(t), e(t) \in C(R, R+)$ are bounded and periodic functions and $m \in(0,1]$ is a constant. Some sufficient conditions are obtained for the permanence, attractivity, and existence of the positive periodic solution of the system (6).

On the other hand, ecosystems in the real world are continuously distributed by unpredictable forces which can result in changes in the biological parameters such as survival rates. Of practical interest in ecology is the question of whether or not an ecosystem can withstand those unpredictable disturbances which persist for a finite period of time. In the language of control variables, we call the disturbance functions as control variables, whereas, the control variables discussed in most literatures are constants or time dependent [8-10]. In recent, there are many works on the feedback controls (see, e.g., [11-19] and the references therein). Furthermore, in recent research on species, dynamics of the Leslie system has important significance; see [20-29] and the references therein for details.

Therefore, Chen and Cao [5] have investigated the following Leslie system with feedback controls:

$$
\begin{array}{r}
\dot{x}_{1}(t)=x_{1}(t)\left[b(t)-a(t) x_{1}(t)\right. \\
\left.-\frac{c(t) x_{1}(t) x_{2}(t)}{h^{2} x_{2}^{2}(t)+x_{1}^{2}(t)}-d(t) u_{1}(t)\right], \\
\dot{x}_{2}(t)=x_{2}(t)\left[g(t)-f(t) \frac{x_{2}(t)}{x_{1}(t)}-p(t) u_{2}(t)\right], \\
\dot{u}_{1}(t)=\alpha_{1}(t)-\beta_{1}(t) u_{1}(t)+\gamma_{1}(t) x_{1}(t), \\
\dot{u}_{2}(t)=\alpha_{2}(t)-\beta_{2}(t) u_{2}(t)+\gamma_{2}(t) x_{2}(t),
\end{array}
$$

where $x_{1}(t)$ and $x_{2}(t)$ denote the density of prey and predator at time $t$, respectively, $u_{i}(t)(i=1,2)$ are control variables, $b, e \in C(R, R)$ are almost periodic functions of $t$, $a, c, d, f, p, \alpha_{i}, \beta_{i}, \gamma_{i} \in C\left(R, R_{+}\right)(i=1,2)$ are all nonnegative almost periodic functions of $t$, and $h^{2}$ is a positive constant denoting the constant of capturing half-saturation.

By using a comparison theorem and constructing a suitable Lyapunov function, they obtained some sufficient conditions for the existence of a unique almost periodic solution and the global attractivity of the solutions of the system (7). By using the comparison and continuation theorems, and based on the coincidence degree and by constructing a suitable Lyapunov function, Wang et al. [30] further obtained some sufficient and necessary conditions for the existence and global attractivity of periodic solutions of the system (7) with the periodic coefficients.

However, to the best of our knowledge, up to now, still no scholar has considered the general nonautonomous Leslie predator-prey system with mutual interference and feedback controls. In this paper, we will consider following the Leslie predator-prey system:

$$
\begin{gathered}
\dot{x}_{1}(t)=x_{1}(t)\left[b(t)-a(t) x_{1}(t)\right. \\
\left.-\frac{c(t) x_{1}^{p-1}(t) x_{2}^{m}(t)}{k+x_{1}^{p}(t)}-d(t) u_{1}(t)\right], \\
\dot{x}_{2}(t)=x_{2}(t)\left[g(t)-f(t) \frac{x_{2}(t)}{x_{1}(t)}-p(t) u_{2}(t)\right], \\
\dot{u}_{1}(t)=\alpha_{1}(t)-\beta_{1}(t) u_{1}(t)+\gamma_{1}(t) x_{1}(t), \\
\dot{u}_{2}(t)=\alpha_{2}(t)-\beta_{2}(t) u_{2}(t)+\gamma_{2}(t) x_{2}(t),
\end{gathered}
$$

where $x_{1}(t)$ and $x_{2}(t)$ denote the density of prey and predator at time $t$, respectively, $u_{i}(t)(i=1,2)$ are control variables, $b(t), a(t), c(t), d(t), g(t), f(t), p(t), \alpha_{i}(t), \beta_{i}(t), \gamma_{i}(t)(i=$ $1,2) \in C\left(R, R_{+}\right), p \geq 1, k>0$ and $m \in(0,1]$ are positive constants.

For the general nonautonomous case, by using differential inequality theory and constructing a suitable Lyapunov functional, we obtain some sufficient conditions which guarantee the permanence and the global attractivity of the system (8). For the periodic case, we obtain some sufficient conditions which guarantee the existence, uniqueness, and stability of a positive periodic solution of the system (8). We would like to mention that the conditions are related to the interference constant $m$.

\section{Preliminaries}

In this section, we state several definitions and lemmas which will be useful in proving the main results of this paper.

Let $R$ and $R^{n}$, respectively, denote the set of all real numbers and the $n$-dimensional real Euclidean space, and $R_{+}^{n}$ denote the nonnegative cone of $R^{n}$. Let $f$ be a continuous bounded function on $R$ and we set $f^{u}=\sup _{t \in R} f(t)$ and $f^{l}=\inf _{t \in R} f(t)$. 
Throughout this paper, we assume that the coefficients of the system (8) satisfy the following conditions:

$$
\begin{gathered}
\min _{i=1,2}\left\{a^{u}, b^{u}, c^{u}, d^{u}, f^{u}, g^{u}, p^{u}, \alpha_{i}^{u}, \beta_{i}^{u}, \gamma_{i}^{u}\right\}>0, \\
\max _{i=1,2}\left\{a^{l}, b^{l}, c^{l}, d^{l}, f^{l}, g^{l}, p^{l}, \alpha_{i}^{l}, \beta_{i}^{l}, \gamma_{i}^{l}\right\}<\infty .
\end{gathered}
$$

Definition 1. If $(\bar{x}(t), \bar{y}(t), \bar{u}(t), \bar{v}(t))^{T}$ is a positive solution of the system $(8)$ and $(x(t), y(t), u(t), v(t))^{T}$ is any positive solution of system $(8)$ satisfying

$$
\begin{array}{ll}
\lim _{t \rightarrow+\infty}|\bar{x}(t)-x(t)|=0, & \lim _{t \rightarrow+\infty}|\bar{y}(t)-y(t)|=0, \\
\lim _{t \rightarrow+\infty}|\bar{u}(t)-u(t)|=0, & \lim _{t \rightarrow+\infty}|\bar{v}(t)-v(t)|=0,
\end{array}
$$

then we say that $(\bar{x}(t), \bar{y}(t), \bar{u}(t), \bar{v}(t))^{T}$ is globally attractive.

Lemma 2 (see [31]). If $a>0, b>0$, and $\dot{x} \geq(\leq) b$-ax, when $t \geq t_{0}$ and $x\left(t_{0}\right)>0$, one has

$$
x(t) \geq(\leq) \frac{b}{a}\left[1+\left(\frac{a x\left(t_{0}\right)}{b}-1\right) e^{-a\left(t-t_{0}\right)}\right]^{-1} .
$$

Lemma 3 (see [31]). If $a>0, b>0$, and $\dot{x} \geq(\leq) x(b-a x)$, when $t \geq t_{0}$ and $x\left(t_{0}\right)>0$, one has

$$
x(t) \geq(\leq) \frac{b}{a}\left[1+\left(\frac{b}{a x\left(t_{0}\right)}-1\right) e^{-b\left(t-t_{0}\right)}\right]^{-1} .
$$

Lemma 4 (see [32]). Iffunction $f$ is nonnegative, integral, and uniformly continuous on $[0,+\infty)$, then

$$
\lim _{t \rightarrow+\infty} f(t)=0
$$

One assumes the following hypothesis holds:

$$
\begin{aligned}
& \left(H_{1}\right) k b^{l}>c^{u} M_{1}^{p-1} M_{2}^{m}+k d^{u} M_{3} ; \\
& \left(H_{2}\right) g^{l}>p^{u} M_{4} \\
& \left(H_{3}\right)\left[s_{2} f(t) / M_{1}-w_{2} \gamma_{2}(t)-w_{1} \gamma_{2}(t)-s_{1} m m_{2}^{m-1} M_{1}^{p-1} c(t) /\right. \\
& \left.\left(k+m_{1}^{p}\right)\right]^{l}>0 \\
& \left(H_{4}\right)\left[w_{1} \beta_{1}(t)-s_{1} d(t)+w_{2} \beta_{1}(t)\right]^{l}>0 ; \\
& \left(H_{5}\right)\left[w_{1} \beta_{2}(t)-s_{2} p(t)+w_{2} \beta_{2}(t)\right]^{l}>0 \text {; } \\
& \left(H_{6}\right) \text { for } 1 \leq p \leq 2 \text {, } \\
& {\left[s_{1} a(t)-w_{1} \gamma_{1}(t)-w_{2} \gamma_{1}(t)+\frac{k(p-1) s_{1} m_{2}^{m} M_{1}^{p-2} c(t)}{\left(k+M_{1}^{p}\right)^{2}}\right.} \\
& \left.-\frac{s_{1} M_{1}^{2 p-2} c(t)}{\left(k+m_{1}^{p}\right)^{2}}-\frac{s_{2} M_{2} f(t)}{m_{1}^{2}}\right]^{l}>0 ;
\end{aligned}
$$

$\left(H_{7}\right)$ for $p>2$,

$$
\left[s_{1} a(t)-w_{1} \gamma_{1}(t)-w_{2} \gamma_{1}(t)+\frac{k(p-1) s_{1} m_{2}^{m} m_{1}^{p-2} c(t)}{\left(k+M_{1}^{p}\right)^{2}}\right.
$$$$
\left.-\frac{s_{1} M_{1}^{2 p-2} c(t)}{\left(k+m_{1}^{p}\right)^{2}}-\frac{s_{2} M_{2} f(t)}{m_{1}^{2}}\right]^{l}>0
$$

where $s_{i}$ and $w_{i}(i=1,2)$ are positive constants.

For any given the initial conditions of the system (8), $x_{i 0}=$ $x_{i}\left(t_{0}\right)>0, u_{i 0}=u_{i}\left(t_{0}\right)>0(i=1,2)$, it is not difficult to see that the corresponding solution $\left(x_{1}(t), x_{2}(t), u_{1}(t), u_{2}(t)\right)^{T}$ satisfies $x_{i}(t)>0, u_{i}(t)>0(i=1,2)$, for $t \geq t_{0}$.

\section{General Nonautonomous Case}

In this section, we will explore the dynamics of the system (8) and present some results including the permanence and the global attractivity of the system.

Theorem 5. Suppose that system (8) satisfies the assumptions $\left(H_{1}\right)$ and $\left(H_{2}\right)$. Then system $(8)$ is permanent; that is, any positive solution $\left(x_{1}(t), x_{2}(t), u_{1}(t), u_{2}(t)\right)^{T}$ of the system (8) satisfies

$$
\begin{gathered}
0<m_{i} \leq \liminf _{t \rightarrow+\infty} x_{i}(t) \leq \limsup _{t \rightarrow+\infty} x_{i}(t) \leq M_{i}, \\
0<m_{2+i} \leq \liminf _{t \rightarrow+\infty} u_{i}(t) \leq \lim _{t \rightarrow+\infty} \sup _{i}(t) \leq M_{2+i}, \quad i=1,2,
\end{gathered}
$$

where

$$
\begin{aligned}
& M_{1}=\frac{b^{u}}{a^{l}}, \quad M_{2}=\frac{g^{u} b^{u}}{f^{l} a^{l}}, \\
& M_{3}=\frac{a^{l} \alpha_{1}^{u}+\gamma_{1}^{u} b^{u}}{a^{l} \beta_{1}^{l}}, \quad M_{4}=\frac{f^{l} a^{l} \alpha_{2}^{u}+\gamma_{2}^{u} g^{u} b^{u}}{f^{l} a^{l} \beta_{2}^{l}}
\end{aligned}
$$

with

$$
\begin{gathered}
m_{1}=\frac{k b^{l}-c^{u} M_{1}^{p-1} M_{2}^{m}-k d^{u} M_{3}}{k a^{u}}, \\
m_{2}=\frac{m_{1}\left(g^{l}-p^{u} M_{4}\right)}{f^{u}}, \\
m_{3}=\frac{\alpha_{1}^{l}+\gamma_{1}^{l} m_{1}}{\beta_{1}^{u}}, \quad m_{4}=\frac{\alpha_{2}^{l}+\gamma_{2}^{l} m_{2}}{\beta_{2}^{u}} .
\end{gathered}
$$

Proof. From the first equation of the system (8), it follows that

$$
\dot{x}_{1}(t) \leq x_{1}(t)\left(b^{u}-a^{l} x_{1}(t)\right) \text {. }
$$

Applying Lemma 3 to (19), for a small enough positive constant $\epsilon>0$, there exists a $T_{1}>0$ enough large such that

$$
x_{1}(t) \leq \frac{b^{u}}{a^{l}}+\epsilon=M_{1}+\epsilon, \quad \forall t>T_{1} .
$$


From the second equation of the system (8) and (20), it follows that, for $t>T_{1}$,

$$
\dot{x}_{2}(t) \leq x_{2}(t)\left[g^{u}-f^{l} \frac{x_{2}(t)}{\left(b^{u} / a^{l}+\epsilon\right)}\right] .
$$

Setting $\epsilon \rightarrow 0$ in inequality (21), we get

$$
\dot{x}_{2}(t) \leq x_{2}(t)\left[g^{u}-f^{l} a^{l} \frac{x_{2}(t)}{b^{u}}\right] .
$$

For any $\epsilon>0$, it follows from (22) and Lemma 3 that there exists a $T_{2}>T_{1}$ such that, for ant $t>T_{2}$,

$$
x_{2}(t) \leq \frac{g^{u} b^{u}}{f^{l} a^{l}}+\epsilon=M_{2}+\epsilon .
$$

By the third equation of system (8) and (20), we obtain

$$
\dot{u}_{1}(t) \leq \alpha_{1}^{u}-\beta_{1}^{l} u_{1}(t)+\gamma_{1}^{u}\left(M_{1}+\epsilon\right) .
$$

Letting $\epsilon \rightarrow 0$ in inequality (24), we get

$$
\dot{u}_{1}(t) \leq \alpha_{1}^{u}+\gamma_{1}^{u} M_{1}-\beta_{1}^{l} u_{1}(t) .
$$

For any $\epsilon>0$, it follows from (25) and Lemma 2 that there exists a $T_{3}>T_{2}$ such that, for any $t>T_{3}$,

$$
u_{1}(t) \leq \frac{a^{l} \alpha_{1}^{u}+\gamma_{1}^{u} b^{u}}{a^{l} \beta_{1}^{l}}+\epsilon=M_{3}+\epsilon
$$

By the forth equation of system (8) and (23), we have

$$
\dot{u}_{2}(t) \leq \alpha_{2}^{u}-\beta_{2}^{l} u_{2}(t)+\gamma_{2}^{u}\left(M_{2}+\epsilon\right) \text {. }
$$

Setting $\epsilon \rightarrow 0$ in inequality (27), we obtain

$$
\dot{u}_{2}(t) \leq \alpha_{2}^{u}-\beta_{2}^{l} u_{2}(t)+\gamma_{2}^{u} M_{2} \text {. }
$$

For any $\epsilon>0$, it follows from (28) and Lemma 2 that there exists a $T_{4}>T_{3}$ such that, for any $t>T_{4}$,

$$
u_{2}(t) \leq \frac{f^{l} a^{l} \alpha_{2}^{u}+\gamma_{2}^{u} g^{u} b^{u}}{\beta_{2}^{l} f^{l} a^{l}}+\epsilon=M_{4}+\epsilon .
$$

By the first equation of system (8), (20), (23), and (26), we obtain

$$
\begin{aligned}
\dot{x}_{1}(t) \geq & x_{1}(t) \\
\times & {\left[b^{l}-a^{u} x_{1}(t)-\frac{c^{u}\left(M_{1}+\epsilon\right)^{p-1}\left(M_{2}+\epsilon\right)^{m}}{k}\right.} \\
& \left.-d^{u}\left(M_{3}+\epsilon\right)\right] .
\end{aligned}
$$

Taking $\epsilon \rightarrow 0$ in inequality (30), we have

$$
\dot{x}_{1}(t) \geq x_{1}(t)\left[b^{l}-\frac{c^{u} M_{1}^{p-1} M_{2}^{m}}{k}-d^{u} M_{3}-a^{u} x_{1}(t)\right] \text {. }
$$

In view of condition $\left(H_{1}\right)$ and Lemma 3, for any $\epsilon>0$, it follows from (28) that there exists a $T_{5}>T_{4}$ such that, for any $t>T_{5}$,

$$
x_{1}(t) \geq \frac{k b^{l}-c^{u} M_{1}^{p-1} M_{2}^{m}-k d^{u} M_{3}}{k a^{u}}-\epsilon=m_{1}-\epsilon .
$$

From the second equation of the system (8), (29), and (32), we have

$$
\dot{x}_{2}(t) \geq x_{2}(t)\left[g^{l}-f^{u} \frac{x_{2}(t)}{m_{1}-\epsilon}-p^{u}\left(M_{4}+\epsilon\right)\right] .
$$

Setting $\epsilon \rightarrow 0$ in inequality (33), we have

$$
\dot{x}_{2}(t) \geq x_{2}(t)\left[g^{l}-p^{u} M_{4}-f^{u} \frac{x_{2}(t)}{m_{1}}\right] \text {. }
$$

In view of condition $\left(\mathrm{H}_{2}\right)$ and Lemma 3, for any $\epsilon>0$, (34) shows that there exists a $T_{6}>T_{5}$ such that, for any $t>T_{6}$,

$$
x_{2}(t) \geq \frac{m_{1}\left(g^{l}-p^{u} M_{4}\right)}{f^{u}}-\epsilon=m_{2}-\epsilon .
$$

By the third equation of system (8) and (32), we obtain

$$
u_{1}(t) \geq \alpha_{1}^{l}-\beta_{1}^{u} u_{1}(t)+\gamma_{1}^{l}\left(m_{1}-\epsilon\right) .
$$

Letting $\epsilon \rightarrow 0$ in inequality (36), we get

$$
u_{1}(t) \geq \alpha_{1}^{l}+\gamma_{1}^{l} m_{1}-\beta_{1}^{u} u_{1}(t) \text {. }
$$

For any $\epsilon>0$, it follows from (37) and Lemma 2 that there exists a $T_{7}>T_{6}$ such that, for any $t>T_{7}$,

$$
u_{1}(t) \geq \frac{\alpha_{1}^{l}+\gamma_{1}^{l} m_{1}}{\beta_{1}^{u}}-\epsilon=m_{3}-\epsilon .
$$

By the forth equation of system (8) and (35), we have

$$
u_{2}(t) \geq \alpha_{2}^{l}-\beta_{2}^{u} u_{2}(t)+\gamma_{2}^{l}\left(m_{2}-\epsilon\right) .
$$

Taking $\epsilon \rightarrow 0$ in inequality (39), we get

$$
u_{2}(t) \geq \alpha_{2}^{l}-\beta_{2}^{u} u_{2}(t)+\gamma_{2}^{l} m_{2}
$$

For any $\epsilon>0$, by Lemma 2 and (40), there exists a $T_{8}>T_{7}$ such that, for any $t>T_{8}$,

$$
u_{2}(t) \geq \frac{\alpha_{2}^{l}+\gamma_{2}^{l} m_{2}}{\beta_{2}^{u}}-\epsilon=m_{4}-\epsilon .
$$

Under the conditions $\left(H_{1}\right)$ and $\left(H_{2}\right)$, it follows from (20), (23), (26), (29), (32), (35), (38), and (41) that the system (8) is permanent. This completes the proof.

In the following, by constructing a suitable Lyapunov functional, we get the sufficient conditions for the globally attractive solution for the system (8). 
Theorem 6. Suppose that system (8) satisfies the assumptions $\left(H_{1}\right)-\left(H_{6}\right)$ or $\left(H_{1}\right)-\left(H_{5}\right)$ and $\left(H_{7}\right)$. Then for any positive solution $z(t)=\left(x_{1}(t), x_{2}(t), u_{1}(t), u_{2}(t)\right)^{T}$ and $\bar{z}(t)=$ $\left(y_{1}(t), y_{2}(t), v_{1}(t), v_{2}(t)\right)^{T}$ of the system $(8)$, one has

$$
\lim _{t \rightarrow+\infty}|z(t)-\bar{z}(t)|=0 \text {. }
$$

Proof. Let $z(t)=\left(x_{1}(t), x_{2}(t), u_{1}(t), u_{2}(t)\right)^{T}$ and $\bar{z}(t)=$ $\left(y_{1}(t), y_{2}(t), v_{1}(t), v_{2}(t)\right)^{T}$ be any positive solutions of the system (8). It follows from Theorem 5 that there exists $T_{9}>0$ such that

$$
m_{i} \leq x_{i}(t), \quad y_{i}(t) \leq M_{i},
$$

$$
m_{2+i} \leq u_{i}(t), \quad v_{i}(t) \leq M_{2+i} \quad(i=1,2) \text { for } t>T_{9} .
$$

Consider the following functional:

$$
V(t)=\sum_{i=1}^{2}\left[s_{i}\left|\ln x_{i}(t)-\ln y_{i}(t)\right|+w_{i} \sum_{i=1}^{2}\left|u_{i}(t)-v_{i}(t)\right|\right] .
$$

Calculating the upper right derivative of $V(t)$ along the solution of the system (8), it follows that

$$
\begin{aligned}
& V^{+}(t) \\
& =s_{1} \operatorname{sgn}\left(x_{1}(t)-y_{1}(t)\right)\left[\frac{\dot{x}_{1}(t)}{x_{1}(t)}-\frac{\dot{y}_{1}(t)}{y_{1}(t)}\right] \\
& +w_{1} \operatorname{sgn}\left(u_{1}(t)-v_{1}(t)\right)\left(\dot{u}_{1}(t)-\dot{v}_{1}(t)\right) \\
& +w_{1} \operatorname{sgn}\left(u_{2}(t)-v_{2}(t)\right)\left(\dot{u}_{2}(t)-\dot{v}_{2}(t)\right) \\
& +s_{2} \operatorname{sgn}\left(x_{2}(t)-y_{2}(t)\right)\left[\frac{\dot{x}_{2}(t)}{x_{2}(t)}-\frac{\dot{y}_{2}(t)}{y_{2}(t)}\right] \\
& +w_{2} \operatorname{sgn}\left(u_{1}(t)-v_{1}(t)\right)\left(\dot{u}_{1}(t)-\dot{v}_{1}(t)\right) \\
& +w_{2} \operatorname{sgn}\left(u_{2}(t)-v_{2}(t)\right)\left(\dot{u}_{2}(t)-\dot{v}_{2}(t)\right) \\
& \leq-s_{1} a(t)\left|x_{1}(t)-y_{1}(t)\right|+s_{1} d(t)\left|u_{1}(t)-v_{1}(t)\right| \\
& -w_{1} \beta_{1}(t)\left|u_{1}(t)-v_{1}(t)\right|+w_{1} \gamma_{1}(t)\left|x_{1}(t)-y_{1}(t)\right| \\
& -w_{1} \beta_{2}(t)\left|u_{2}(t)-v_{2}(t)\right|+w_{1} \gamma_{2}(t)\left|x_{2}(t)-y_{2}(t)\right| \\
& +s_{2} p(t)\left|u_{2}(t)-v_{2}(t)\right|-w_{2} \beta_{1}(t)\left|u_{1}(t)-v_{1}(t)\right| \\
& +w_{2} \gamma_{1}(t)\left|x_{1}(t)-y_{1}(t)\right|-w_{2} \beta_{2}(t)\left|u_{2}(t)-v_{2}(t)\right| \\
& +w_{2} \gamma_{2}(t)\left|x_{2}(t)-y_{2}(t)\right|-s_{1} c(t) \operatorname{sgn}\left(x_{1}(t)-y_{1}(t)\right) \\
& \times\left[\frac{x_{1}^{p-1}(t) x_{2}^{m}(t)}{k+x_{1}^{p}(t)}-\frac{y_{1}^{p-1}(t) y_{2}^{m}(t)}{k+y_{1}^{p}(t)}\right] \\
& -s_{2} f(t) \operatorname{sgn}\left(x_{2}(t)-y_{2}(t)\right)\left[\frac{x_{2}(t)}{x_{1}(t)}-\frac{y_{2}(t)}{y_{1}(t)}\right] \text {, } \\
& -s_{1} c(t) \operatorname{sgn}\left(x_{1}(t)-y_{1}(t)\right) \\
& \times\left[\frac{x_{1}^{p-1}(t) x_{2}^{m}(t)}{k+x_{1}^{p}(t)}-\frac{y_{1}^{p-1}(t) y_{2}^{m}(t)}{k+y_{1}^{p}(t)}\right]
\end{aligned}
$$

$$
\begin{aligned}
& =-s_{1} c(t) \operatorname{sgn}\left(x_{1}(t)-y_{1}(t)\right) \\
& \times\left[\frac{x_{1}^{p-1}(t) x_{2}^{m}(t)}{k+x_{1}^{p}(t)}-\frac{x_{1}^{p-1}(t) y_{2}^{m}(t)}{k+x_{1}^{p}(t)}\right. \\
& \left.+\frac{x_{1}^{p-1}(t) y_{2}^{m}(t)}{k+x_{1}^{p}(t)}-\frac{y_{1}^{p-1}(t) y_{2}^{m}(t)}{k+y_{1}^{p}(t)}\right] \\
& \leq s_{1} c(t) \frac{x_{1}^{p-1}(t)}{k+x_{1}^{p}(t)}\left|x_{2}^{m}(t)-y_{2}^{m}(t)\right| \\
& -\frac{k s_{1} c(t) y_{2}^{m}(t)}{\left(k+x_{1}^{p}(t)\right)\left(k+y_{1}^{p}(t)\right)}\left|x_{1}^{p-1}(t)-y_{1}^{p-1}(t)\right| \\
& +s_{1} c(t) \frac{x_{1}^{p-1}(t) y_{1}^{p-1}(t)}{\left(k+x_{1}^{p}(t)\right)\left(k+y_{1}^{p}(t)\right)}\left|x_{1}(t)-y_{1}(t)\right| .
\end{aligned}
$$

By the mean value theorem and Theorem 5 , in view of $0<$ $m \leq 1$, for $t>T_{9}$, we have

$$
\begin{array}{r}
\left|x_{2}^{m}(t)-y_{2}^{m}(t)\right| \leq m m_{2}^{m-1}\left|x_{2}(t)-y_{2}(t)\right|, \\
\left|x_{1}^{p-1}(t)-y_{1}^{p-1}(t)\right| \geq(p-1) M_{1}^{p-2}\left|x_{1}(t)-y_{1}(t)\right| \\
\text { when } 1 \leq p \leq 2, \\
\left|x_{1}^{p-1}(t)-y_{1}^{p-1}(t)\right| \geq(p-1) m_{1}^{p-2}\left|x_{1}(t)-y_{1}(t)\right| \\
\text { when } p>2 .
\end{array}
$$

Note that

$$
\begin{aligned}
-s_{2} f( & t) \operatorname{sgn}\left(x_{2}(t)-y_{2}(t)\right)\left[\frac{x_{2}(t)}{x_{1}(t)}-\frac{y_{2}(t)}{y_{1}(t)}\right] \\
= & -s_{2} f(t) \operatorname{sgn}\left(x_{2}(t)-y_{2}(t)\right) \\
& \times\left[\frac{x_{2}(t)}{x_{1}(t)}-\frac{y_{2}(t)}{x_{1}(t)}+\frac{y_{2}(t)}{x_{1}(t)}-\frac{y_{2}(t)}{y_{1}(t)}\right] \\
\leq & \frac{-s_{2} f(t)}{x_{1}(t)}\left|x_{2}(t)-y_{2}(t)\right| \\
& +\frac{s_{2} f(t) y_{2}(t)}{x_{1}(t) y_{1}(t)}\left|x_{1}(t)-y_{1}(t)\right| .
\end{aligned}
$$

When $1 \leq p \leq 2$, according to (45), (46), (47), and (49), it follows that, for $t>T_{9}$,

$V^{+}(t)$

$$
\begin{aligned}
& \leq-\left[s_{1} a(t)-w_{1} \gamma_{1}(t)-w_{2} \gamma_{1}(t)\right. \\
& \quad+\frac{k(p-1) s_{1} M_{1}^{p-2} c(t) y_{2}^{m}(t)}{\left(k+x_{1}^{p}(t)\right)\left(k+y_{1}^{p}(t)\right)} \\
& \left.\quad-\frac{s_{1} c(t) x_{1}^{p-1}(t) y_{1}^{p-1}(t)}{\left(k+x_{1}^{p}(t)\right)\left(k+y_{1}^{p}(t)\right)}-\frac{s_{2} f(t) y_{2}(t)}{x_{1}(t) y_{1}(t)}\right]
\end{aligned}
$$




$$
\begin{aligned}
& \times\left|x_{1}(t)-y_{1}(t)\right| \\
- & {\left[\frac{s_{2} f(t)}{x_{1}(t)}-w_{2} \gamma_{2}(t)-w_{1} \gamma_{2}(t)-\frac{s_{1} m m_{2}^{m-1} c(t) x_{1}^{p-1}(t)}{k+x_{1}^{p}(t)}\right] } \\
\times & \left|x_{2}(t)-y_{2}(t)\right| \\
- & {\left[w_{1} \beta_{1}(t)-s_{1} d(t)+w_{2} \beta_{1}(t)\right]\left|u_{1}(t)-v_{1}(t)\right| } \\
- & {\left[w_{1} \beta_{2}(t)-s_{2} p(t)+w_{2} \beta_{2}(t)\right]\left|u_{2}(t)-v_{2}(t)\right| } \\
\leq & -\left[s_{1} a(t)-w_{1} \gamma_{1}(t)-w_{2} \gamma_{1}(t)\right. \\
& \quad+\frac{k(p-1) s_{1} m_{2}^{m} M_{1}^{p-2} c(t)}{\left(k+M_{1}^{p}\right)^{2}}-\frac{s_{1} M_{1}^{2 p-2} c(t)}{\left(k+m_{1}^{p}\right)^{2}} \\
& \left.-\frac{s_{2} M_{2} f(t)}{m_{1}^{2}}\right]\left|x_{1}(t)-y_{1}(t)\right| \\
& {\left[\frac{s_{2} f(t)}{M_{1}}-w_{2} \gamma_{2}(t)-w_{1} \gamma_{2}(t)-\frac{s_{1} m m_{2}^{m-1} M_{1}^{p-1} c(t)}{k+m_{1}^{p}}\right] } \\
\times & \left|x_{2}(t)-y_{2}(t)\right| \\
- & {\left[w_{1} \beta_{1}(t)-s_{1} d(t)+w_{2} \beta_{1}(t)\right]\left|u_{1}(t)-v_{1}(t)\right| } \\
- & {\left[w_{1} \beta_{2}(t)-s_{2} p(t)+w_{2} \beta_{2}(t)\right]\left|u_{2}(t)-v_{2}(t)\right| . }
\end{aligned}
$$

When $p>2$, according to (45), (46), (48), and (49), for $t>T_{9}$, we have

$$
\begin{aligned}
& V^{+}(t) \\
& \leq-\left[s_{1} a(t)-w_{1} \gamma_{1}(t)-w_{2} \gamma_{1}(t)\right. \\
& +\frac{k(p-1) s_{1} m_{1}^{p-2} c(t) y_{2}^{m}(t)}{\left(k+x_{1}^{p}(t)\right)\left(k+y_{1}^{p}(t)\right)} \\
& \left.-\frac{s_{1} c(t) x_{1}^{p-1}(t) y_{1}^{p-1}(t)}{\left(k+x_{1}^{p}(t)\right)\left(k+y_{1}^{p}(t)\right)}-\frac{s_{2} f(t) y_{2}(t)}{x_{1}(t) y_{1}(t)}\right] \\
& \times\left|x_{1}(t)-y_{1}(t)\right| \\
& -\left[\frac{s_{2} f(t)}{x_{1}(t)}-w_{2} \gamma_{2}(t)-w_{1} \gamma_{2}(t)-\frac{s_{1} m m_{2}^{m-1} c(t) x_{1}^{p-1}(t)}{k+x_{1}^{p}(t)}\right] \\
& \times\left|x_{2}(t)-y_{2}(t)\right| \\
& \text { - }\left[w_{1} \beta_{1}(t)-s_{1} d(t)+w_{2} \beta_{1}(t)\right]\left|u_{1}(t)-v_{1}(t)\right| \\
& -\left[w_{1} \beta_{2}(t)-s_{2} p(t)+w_{2} \beta_{2}(t)\right]\left|u_{2}(t)-v_{2}(t)\right|
\end{aligned}
$$

$$
\begin{aligned}
\leq- & {\left[s_{1} a(t)-w_{1} \gamma_{1}(t)-w_{2} \gamma_{1}(t)\right.} \\
& +\frac{k(p-1) s_{1} m_{2}^{m} m_{1}^{p-2} c(t)}{\left(k+M_{1}^{p}\right)^{2}} \\
& \left.\quad-\frac{s_{1} M_{1}^{2 p-2} c(t)}{\left(k+m_{1}^{p}\right)^{2}}-\frac{s_{2} M_{2} f(t)}{m_{1}^{2}}\right]\left|x_{1}(t)-y_{1}(t)\right| \\
- & {\left[\frac{s_{2} f(t)}{M_{1}}-w_{2} \gamma_{2}(t)-w_{1} \gamma_{2}(t)-\frac{s_{1} m m_{2}^{m-1} M_{1}^{p-1} c(t)}{k+m_{1}^{p}}\right] } \\
\times & \left|x_{2}(t)-y_{2}(t)\right| \\
- & {\left[w_{1} \beta_{1}(t)-s_{1} d(t)+w_{2} \beta_{1}(t)\right]\left|u_{1}(t)-v_{1}(t)\right| } \\
- & {\left[w_{1} \beta_{2}(t)-s_{2} p(t)+w_{2} \beta_{2}(t)\right]\left|u_{2}(t)-v_{2}(t)\right| . }
\end{aligned}
$$

When $1 \leq p \leq 2$, it follows from (50) and assumptions $\left(H_{1}\right)-$ $-\left(H_{6}\right)$ that there exist four positive constants $\delta_{j}(j=1,2,3,4)$ such that

$$
V^{+}(t) \leq-\sum_{i=1}^{2} \delta_{i}\left|x_{i}(t)-y_{i}(t)\right|-\sum_{i=1}^{2} \delta_{i+2}\left|u_{i}(t)-v_{i}(t)\right|,
$$

$$
\forall t>T_{9} \text {, }
$$

which shows that $V(t)$ is nonincreasing on $\left[T_{9},+\infty\right)$. Integrating both sides of $(52)$ on $\left[T_{9}, t\right)$, we get

$$
\begin{aligned}
V(t) & +\sum_{i=1}^{2} \delta_{i} \int_{T_{9}}^{t}\left|x_{i}(t)-y_{i}(t)\right| d t \\
& +\sum_{i=1}^{2} \delta_{i+2} \int_{T_{9}}^{t}\left|u_{i}(t)-v_{i}(t)\right| d t \leq V\left(T_{11}\right)<+\infty .
\end{aligned}
$$

This implies that

$$
\left|x_{i}(t)-y_{i}(t)\right|,\left|u_{i}(t)-v_{i}(t)\right| \in L^{1}\left(\left[T_{9},+\infty\right)\right), \quad i=1,2 .
$$

By Lemma 4, we have

$$
\lim _{t \rightarrow+\infty}\left|x_{i}(t)-y_{i}(t)\right|=0, \quad \lim _{t \rightarrow+\infty}\left|u_{i}(t)-v_{i}(t)\right|=0
$$

for $i=1,2$, and so

$$
\lim _{t \rightarrow+\infty}|z(t)-\bar{z}(t)|=0 .
$$

When $p>2$, by similar way, from (51) and assumptions $\left(H_{1}\right)-$ $\left(H_{5}\right)$ and $\left(H_{7}\right)$, we can show that

$$
\lim _{t \rightarrow+\infty}|z(t)-\bar{z}(t)|=0 .
$$

This completes the proof. 


\section{Periodic Case}

To this end, in order to get the existence and uniqueness of a positive periodic solution of the system (8), we further assume that system (8) satisfies the following condition.

$\left(H_{8}\right) b(t), a(t), c(t), d(t), g(t), f(t), p(t), \alpha_{i}(t), \beta_{i}(t), \gamma_{i}(t) \in$ $C(R, R+),(i=1,2)$ are all continuous, real-valued positive $\omega$-periodic functions; $\omega>0$ is a constant.

Let $S=\left\{\left(x_{1}(t), x_{2}(t), u_{1}(t), u_{2}(t)\right)^{T} \in R_{+}^{4} \quad \mid\right.$ $m_{1} \leq x_{1}(t) \leq M_{1}, m_{2} \leq x_{2}(t) \leq M_{2}, m_{3} \leq$ $\left.u_{1}(t) \leq M_{3}, m_{4} \leq u_{2}(t) \leq M_{4}\right\}$. Let $Z\left(t, Z_{0}\right)=$ $\left(x_{1}\left(t, Z_{0}\right), x_{2}\left(t, Z_{0}\right), u_{1}\left(t, Z_{0}\right), u_{2}\left(t, Z_{0}\right)\right)^{T}$. for $t \geq t_{0}$ be the uniqueness solution of the system (8) with the initial condition $Z_{0}=\left(x_{1}\left(t_{0}\right), x_{2}\left(t_{0}\right), u_{1}\left(t_{0}\right), u_{2}\left(t_{0}\right)\right)^{T}$. By Theorem 5 , there exists a $T_{0}>0$ such that $\left(x_{1}(t), x_{2}(t), u_{1}(t), u_{2}(t)\right)^{T} \in S$, for $t \geq T_{0}$.

Since $x_{1}\left(t, Z_{0}\right)$ is continuous on $\left[t_{0}, T_{0}\right]$, there exist two positive constants $m_{1}^{*}$ and $M_{1}^{*}$ such that $m_{1}^{*} \leq x_{1}\left(t, Z_{0}\right) \leq M_{1}^{*}$ for $t \in\left[t_{0}, T_{0}\right]$. Let $l_{1}=\min \left\{m_{1}^{*}, m_{1}\right\}$ and $L_{1}=\max \left\{M_{1}^{*}, M_{1}\right\}$. Then $l_{1} \leq x_{1}\left(t, Z_{0}\right) \leq L_{1}$, for $t>t_{0}$. Similarly, we can find positive constants $l_{2}$ and $L_{2}$ such that $l_{2} \leq x_{2}\left(t, Z_{0}\right) \leq L_{2}$ and positive constants $l_{i}$ and $L_{i}$ such that $l_{i} \leq u_{i-2}\left(t, Z_{0}\right) \leq L_{i}$ $(i=3,4)$ for $t \geq t_{0}$. Let $S^{*}=\left\{\left(x_{1}(t), x_{2}(t), u_{1}(t), u_{2}(t)\right)^{T} \in\right.$ $R_{+}^{4} \mid l_{1} \leq x_{1}(t) \leq L_{1}, l_{2} \leq x_{2}(t) \leq L_{2}, l_{3} \leq u_{1}(t) \leq L_{3}$, $\left.l_{4} \leq u_{2}(t) \leq L_{4}\right\}$. Therefore, we have $Z\left(t, Z_{0}\right) \in S^{*}$, for $t \geq t_{0}$. Define the Poincare mapping $A: R_{+}^{4} \rightarrow R_{+}^{4}$ as follows:

$$
A\left(Z_{0}\right)=Z\left(\omega, Z_{0}\right) \text {, }
$$

where $\omega$ is defined in assumption $\left(H_{8}\right)$.

It is easy to see that the existence of periodic solution in (8) with assumptions $\left(H_{8}\right)$ is equal to prove that the mapping $A$ has at least one fixed point.

Theorem 7. If the assumptions $\left(H_{1}\right),\left(H_{2}\right)$, and $\left(H_{8}\right)$ hold, system (8) has at least one positive $\omega$-periodic solution.

Proof. According to discussion above, we obtain $A\left(S^{*}\right) \subset S^{*}$. And we can get that the mapping $A$ is continuous by the theory of ordinary differential equation. It is obvious that $S^{*}$ is a closed and convex set. Therefore, $A$ has at least one fixed point by Brouwer fixed point theorem. That is to say, if system (8) satisfies $\left(H_{1}\right),\left(H_{2}\right)$, and $\left(H_{8}\right)$, system $(8)$ has at least one positive $\omega$-periodic solution. This completes the proof.

Finally, some sufficient conditions are obtained for the uniqueness of the positive $\omega$-periodic solution for the system (8).

Theorem 8. If system (8) satisfies $\left(H_{1}\right)-\left(H_{6}\right)$ and $\left(H_{8}\right)$ or $\left(H_{1}\right)-\left(H_{5}\right),\left(H_{7}\right)$ and $\left(H_{8}\right)$, system $(8)$ has only one positive $\omega$ periodic solution which is globally attractive.

Proof. If system (8) satisfies $\left(H_{1}\right)-\left(H_{6}\right)$ and $\left(H_{8}\right),(8)$ has at least one $\omega$-periodic solution by Theorem 7 . For any two positive $\omega$-periodic solutions $z(t)=\left(x_{1}(t), x_{2}(t), u_{1}(t), u_{2}(t)\right)^{T}$ and $\bar{z}(t)=\left(y_{1}(t), y_{2}(t), v_{1}(t), v_{2}(t)\right)^{T}$ of the system $(8)$. We claim that $z(t) \equiv \bar{z}(t)$, for all $t \in[0, \omega]$. Otherwise, there must be at least $\xi \in[0, \omega]$ such that $x_{1}(\xi) \neq y_{1}(\xi)$; that is, $\left|x_{1}(\xi)-y_{1}(\xi)\right|:=\rho>0$. It follows that

$$
\begin{aligned}
\rho & =\lim _{n \rightarrow+\infty}\left|x_{1}(\xi+n \omega)-y_{1}(\xi+n \omega)\right| \\
& =\lim _{t \rightarrow+\infty}\left|x_{1}(t)-y_{1}(t)\right|>0,
\end{aligned}
$$

which contracts with (42). Thus $x_{1}(t) \equiv y_{1}(t)$, for all $t \in$ $[0, \omega]$, hods. Similarly, we can prove that

$$
\begin{array}{r}
x_{2}(t) \equiv y_{2}(t), \quad u_{1}(t) \equiv v_{1}(t), \quad u_{2}(t) \equiv v_{2}(t), \\
\forall t \in[0, \omega] .
\end{array}
$$

Therefore, the uniqueness of the periodic solution of the system (8) is obtained. By Theorem 6, if system (8) satisfies $\left(H_{1}\right)-\left(H_{6}\right)$ and $\left(H_{8}\right),(8)$ has only one positive $\omega$-periodic solution which is globally attractive. Similarly, we can prove that system $(8)$ satisfying $\left(H_{1}\right)-\left(H_{5}\right),\left(H_{7}\right)$, and $\left(H_{8}\right)$ has a unique positive $\omega$-periodic solution which is globally attractive. This completes the proof.

\section{Conflict of Interests}

The authors declare that there is no conflict of interests regarding the publication of this paper.

\section{Acknowledgment}

This work was supported by the Key Program of NSFC (Grant no. 70831005) and the National Natural Science Foundation of China (111171237).

\section{References}

[1] P. H. Leslie, "Some further notes on the use of matrices in population mathematics," Biometrika, vol. 35, pp. 213-245, 1948.

[2] F. Chen, "Permanence in nonautonomous multi-species predator-prey system with feedback controls," Applied Mathematics and Computation, vol. 173, no. 2, pp. 694-704, 2006.

[3] R. E. Kooij and A. Zegeling, "Qualitative properties of twodimensional predator-prey systems," Nonlinear Analysis: Theory, Methods and Applications, vol. 29, no. 6, pp. 693-715, 1997.

[4] J. Sugie, R. Kohno, and R. Miyazaki, "On a predator-prey system of rolling type," Proceedings of the American Mathematical Society, vol. 125, no. 7, pp. 2041-2050, 1997.

[5] F. Chen and X. Cao, "Existence of almost periodic solution in a ratio-dependent Leslie system with feedback controls," Journal of Mathematical Analysis and Applications, vol. 341, no. 2, pp. 1399-1412, 2008.

[6] M. P. Hassell, "Density dependence in single-species population," Journal of Animal Ecology, vol. 44, pp. 283-295, 1975.

[7] W. H. Pan, "Qualitative analysis of a Leslie prey-predator system with with mutual interference," Journal of Changsha University, vol. 5, pp. 18-20, 2005 (Chinese).

[8] Y. Kuang, Delay Differential Equations with Application in Population Dynamics, Academic Press, New York, NY, USA, 1993. 
[9] J. Lasalle and S. Lefschetz, Stability by Liapunov's Direct Method, with Applications, Academic Press, New York, NY, USA, 1961.

[10] S. Lefschetz, Stability of Nonlinear Control System, Academic Press, New York, NY, USA, 1965.

[11] M. Fan, K. Wang, P. J. Y. Wong, and R. P. Agarwal, "Periodicity and stability in periodic $n$-species Lotka-Volterra competition system with feedback controls and deviating arguments," Acta Mathematica Sinica, vol. 19, no. 4, pp. 801-822, 2003.

[12] $\mathrm{H}$. Hu, Z. Teng, and H. Jiang, "Permanence of the nonautonomous competitive systems with infinite delay and feedback controls," Nonlinear Analysis: Real World Applications, vol. 10, no. 4, pp. 2420-2433, 2009.

[13] W.-T. Li and L.-L. Wang, "Existence and global attractivity of positive periodic solutions of functional differential equations with feedback control," Journal of Computational and Applied Mathematics, vol. 180, no. 2, pp. 293-309, 2005.

[14] Y. Li and T. Zhang, "Permanence and almost periodic sequence solution for a discrete delay logistic equation with feedback control," Nonlinear Analysis: Real World Applications, vol. 12, no. 3, pp. 1850-1864, 2011.

[15] L. Nie, J. Peng, and Z. Teng, "Permanence and stability in multispecies non-autonomous Lotka-Volterra competitive systems with delays and feedback controls," Mathematical and Computer Modelling, vol. 49, no. 1-2, pp. 295-306, 2009.

[16] P. Weng, "Existence and global stability of positive periodic solution of periodic integrodifferential systems with feedback controls," Computers and Mathematics with Applications, vol. 40, no. 6, pp. 747-759, 2000.

[17] W. Wendi and T. Chunlei, "Dynamics of a delayed population model with feedback control," Australian Mathematical Society $B$, vol. 41, no. 4, pp. 451-457, 2000.

[18] R. Xu and F.-l. Hao, "Global stability of a delayed singlespecies population model with feedback control," Journal of Biomathematics, vol. 20, no. 1, pp. 1-6, 2005.

[19] Z. Zhao, T. Wang, and L. Chen, "Dynamic analysis of a turbidostat model with the feedback control," Communications in Nonlinear Science and Numerical Simulation, vol. 15, no. 4, pp. 163-171, 2010.

[20] M. A. Aziz-Alaoui and M. Daher Okiye, "Boundedness and global stability for a predator-prey model with modified LeslieGower and Holling-type II schemes," Applied Mathematics Letters, vol. 16, no. 7, pp. 1069-1075, 2003.

[21] L. Chen and F. Chen, "Global stability of a Leslie-Gower predator-prey model with feedback controls," Applied Mathematics Letters, vol. 22, no. 9, pp. 1330-1334, 2009.

[22] F. Chen, L. Chen, and X. Xie, "On a Leslie-Gower predator-prey model incorporating a prey refuge," Nonlinear Analysis: Real World Applications, vol. 10, no. 5, pp. 2905-2908, 2009.

[23] Y. Du, R. Peng, and M. Wang, "Effect of a protection zone in the diffusive Leslie predator-prey model," Journal of Differential Equations, vol. 246, no. 10, pp. 3932-3956, 2009.

[24] S. Gakkhar and B. Singh, "Dynamics of modified Leslie-Gowertype prey-predator model with seasonally varying parameters," Chaos, Solitons and Fractals, vol. 27, no. 5, pp. 1239-1255, 2006.

[25] S.-B. Hsu and T.-W. Huang, "Global stability for a class of predator-prey systems," SIAM Journal on Applied Mathematics, vol. 55, no. 3, pp. 763-783, 1995.

[26] Y. Li and D. Xiao, "Bifurcations of a predator-prey system of Holling and Leslie types," Chaos, Solitons and Fractals, vol. 34, no. 2, pp. 606-620, 2007.
[27] T. Saha and C. Chakrabarti, "Dynamical analysis of a delayed ratio-dependent Holling-Tanner predator-prey model," Journal of Mathematical Analysis and Applications, vol. 358, no. 2, pp. 389-402, 2009.

[28] R. Yafia, F. El Adnani, and H. T. Alaoui, "Limit cycle and numerical similations for small and large delays in a predatorprey model with modified Leslie-Gower and Holling-type II schemes," Nonlinear Analysis: Real World Applications, vol. 9, no. 5, pp. 2055-2067, 2008.

[29] S. Yuan and Y. Song, "Stability and Hopf bifurcations in a delayed Leslie-Gower predator-prey system," Journal of Mathematical Analysis and Applications, vol. 355, no. 1, pp. 82-100, 2009.

[30] Q. Wang, J. Zhou, Z. J. Wang, M. M. Ding, and H. Y. Zhang, "Qualitative properties of two-dimensional predatorprey system," Nonlinear Analysis: Real World Applications, vol. 12, pp. 24-33, 2011.

[31] F. Chen, X. Liao, and Z. Huang, "The dynamic behavior of Nspecies cooperation system with continuous time delays and feedback controls," Applied Mathematics and Computation, vol. 181, no. 2, pp. 803-815, 2006.

[32] K. Gopalasamy, Stability and Oscillation in Delay Equation of Population Dynamics, Kluwer Academic Publishers, Dordrecht, Germany, 1992. 


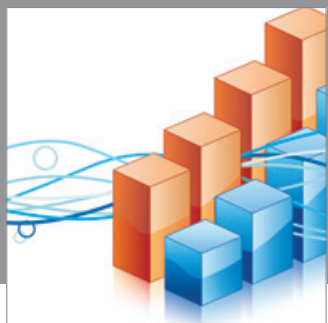

Advances in

Operations Research

mansans

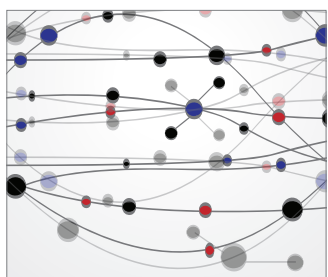

The Scientific World Journal
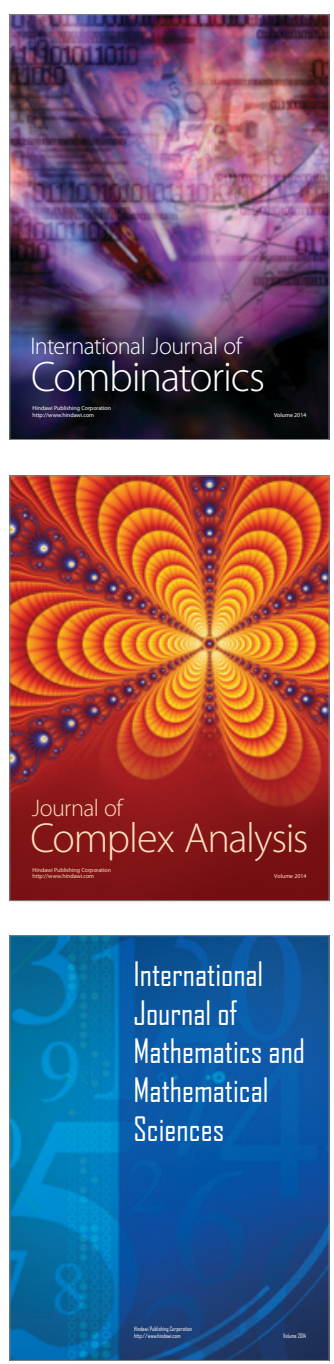
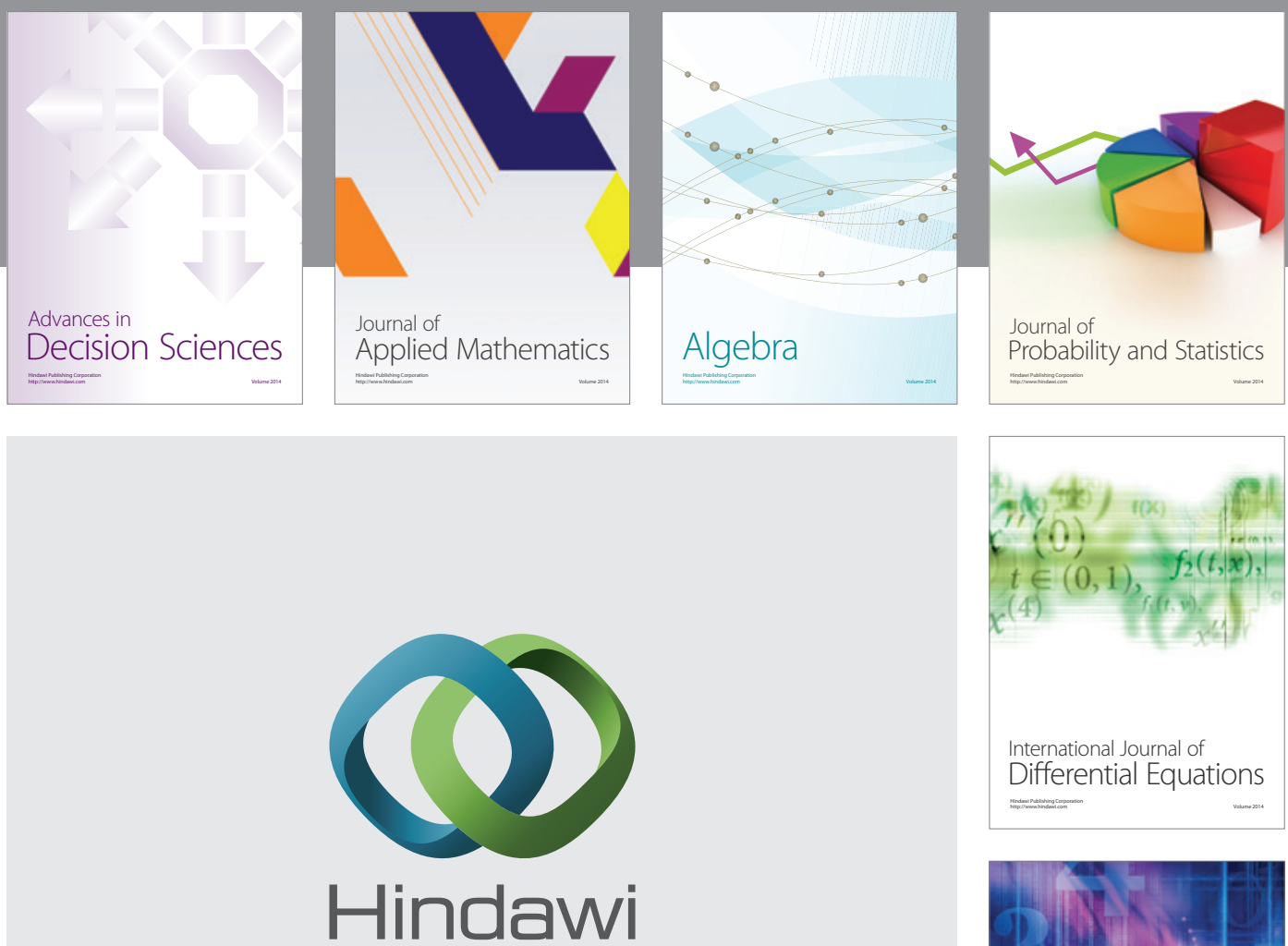

Submit your manuscripts at http://www.hindawi.com
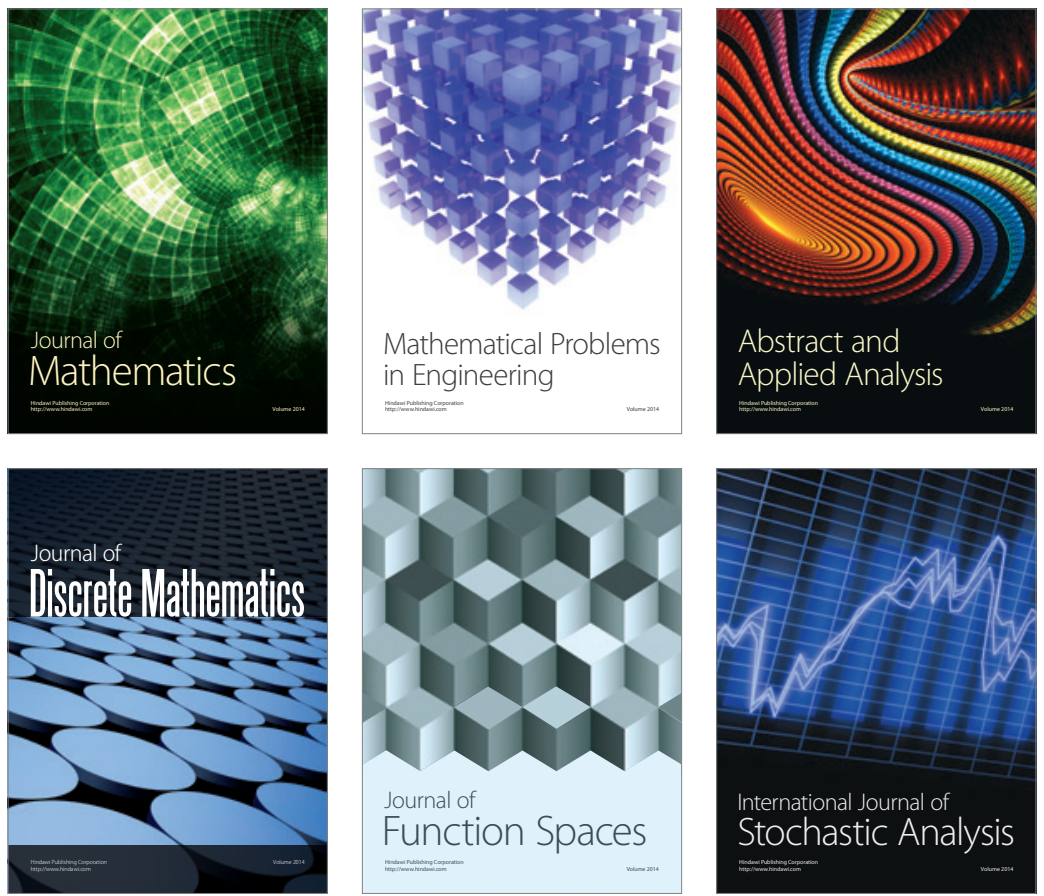

Journal of

Function Spaces

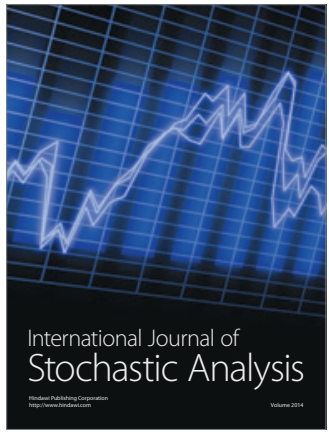

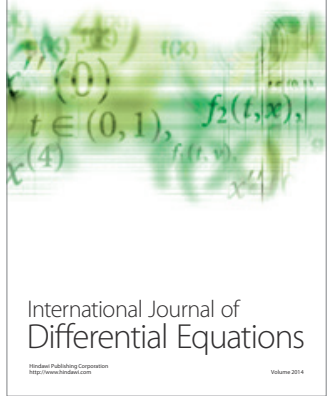
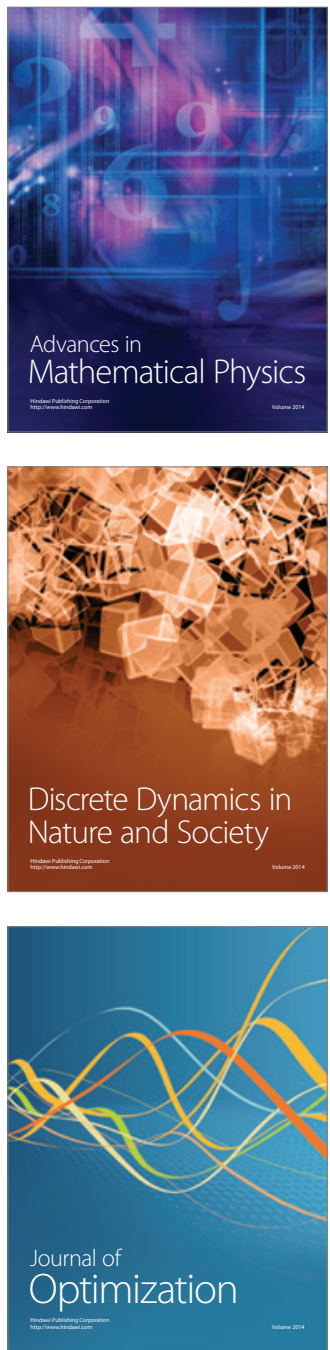